\title{
Post-psychotic depression: Paranoia and the damage done
}

\author{
Steffen Moritz ${ }^{\mathrm{a}, *, 1}$, Stefanie J. Schmidt ${ }^{\mathrm{b}, 1}$, Thies Lüdtke ${ }^{\mathrm{a}, \mathrm{c}}$, Lea-Elena Braunschneider ${ }^{\mathrm{a}}$, Alisa Manske ${ }^{\mathrm{b}}$, \\ Brooke C. Schneider ${ }^{\mathrm{a}, 2}$, Ruth Veckstenstedt ${ }^{\mathrm{a}, 2}$ \\ a University Medical Center Hamburg-Eppendorf, Department of Psychiatry and Psychotherapy, Hamburg, Germany \\ ${ }^{\mathrm{b}}$ Department of Clinical Psychology and Psychotherapy, University of Bern, Bern, Switzerland \\ c Department of Psychology, UiT - The Arctic University of Norway, Tromsø, Norway
}

Keywords:

Depression

Paranoia

Cross-lagged models

Positive and Negative Syndrome Scale (PANSS)

Psychosis Rating Scales (PSYRATS)

\begin{abstract}
A B S T R A C T
To mitigate the often chronic course of schizophrenia and improve functional outcome, researchers are increasingly interested in prodromal states and psychological risk factors that may predict the outbreak of psychotic symptoms, but are also amenable to change. In recent years, depressive symptoms have been proposed as precursors of psychosis and some interventional studies indicate that the amelioration of depressive symptoms and depression-related thinking styles (e.g., worrying) improves positive symptoms, thereby "killing two birds with one stone". Yet, in a prior study, we were unable to find a strong specific predictive role of depression on paranoia over three years, which may have been due to the use of a nonclinical sample with minimal/mild symptom fluctuations. To address this further, in the present study we adopted a similar methodological approach but assessed a large patient sample with a schizophrenia spectrum disorder at three assessment points; baseline $(N=250), 6$ weeks later $(n=207$, $82.8 \%$ retention) and 6 months after baseline $(n=185,74 \%$ retention). Using cross-lagged modeling, we assessed paranoia with the respective items from the Positive and Negative Syndrome Scale (PANSS) and the Psychosis Rating Scales (PSYRATS) delusions subscale. Depression was measured using the Patient Health Questionnaire-9 (PHQ9) and the Calgary Depression Scale for Schizophrenia (CDSS). We could identify a significant pathway from depression to paranoia from baseline to post (negative association) but not from post to follow-up. Paranoia significantly predicted depressive symptoms for both intervals. Our findings do not refute claims that depression may precede or even predict psychosis, but such a linkage does not seem to be ubiquitous.
\end{abstract}

\section{Introduction}

\subsection{Schizophrenia and affective disorders - now and then}

Since the days of Emil Kraepelin, schizophrenia (then called dementia praecox) has been distinguished from affective illness; both disorders were regarded as etiologically separable diseases (Moritz et al., 2019). This view has diminished over the years and it is now acknowledged that depression and schizophrenia can coexist as in schizoaffective disorder or post-psychotic depression. Influential researchers such as Crow (1995) even presume an "Einheitspsychose", that is, a shared vulnerability of the two. According to this view, mania, depression and psychosis are different manifestations of similar vulnerability factors and indeed genetic overlap seems to be high (e.g., Cardno and Owen, 2014; Wray et al., 2018). Approximately $50 \%$ of schizophrenia patients suffer from depressive symptoms that require treatment (Buckley et al., 2009) and even

\footnotetext{
* Corresponding author

E-mail address: moritz@uke.uni-hamburg.de (S. Moritz).

1 Split first authorship.

2 Split senior authorship.
}

more display subclinical symptoms. The prevalence of these symptoms seems to differ across the different phases of the disorder. Upthegrove (2009) has gathered data showing that the prevalence of depression is up to $83 \%$ in high-risk states, up to $5 \%$ in acute cases, and up to $54 \%$ after a psychotic episode. Not surprisingly, depression is among the symptoms patients prefer to be treated first, even before positive symptoms, the classical target of psychiatric treatment (Byrne et al., 2010; Kuhnigk et al., 2012; Moritz et al., 2017a).

\subsection{Depression as a precursor or even risk factor for paranoia}

In the last decades, researchers have begun to explore the dynamics between psychosis and core depressive symptoms (e.g., low mood, lack of drive) or depressive thinking styles (e.g, worry). Evidence for an association between depressive symptoms and psychosis has evolved from different branches of research. The ABC study by Häfner and colleagues (Häfner et al., 2013) was among the first to suggest that depression may be part of the (unspecific) clinical high risk state of psychosis. This study used retrospective assessment, which has severe methodological limitations (e.g., memory biases such as the telescoping effect, attributional biases/search for meaning, locus of control, etc.). To address these 
shortcomings, current research increasingly adopts the experience sampling method (ESM; Myin-Germeys et al., 2018), which allows the detection of prospective associations between precursors and psychotic symptoms in the daily life of participants. ESM studies suggest that core features of depression precede psychotic symptoms, particularly paranoia. Among such precursors are low self-esteem, experiential avoidance (Udachina et al., 2014), and worry (Hartley et al., 2014). In addition, psychotic symptoms are preceded by "negative affect", a term subsuming feelings of sadness and anxiety but also loneliness or tension (Ben-Zeev et al., 2011; Kramer et al., 2014; Lüdtke et al., 2017; So et al., 2018).

Based on these and other findings, an influential model advocated by British researchers (Freeman, 2016; Freeman and Garety, 2014; Garety and Freeman, 2013) ascribes depression and depression-related symptoms a pivotal role for the pathogenesis of paranoia. They propose that emotional biases (i.e., negative beliefs about the self, worrying and interpersonal sensitivity) and sleep problems along with cognitive biases (inflexibility, jumping to conclusions) lead to paranoia. Support for this model stems from evidence that interventions targeted at these alleged risk factors (e.g., mindfulness, CBT for insomnia) decrease paranoid symptoms (Bullock et al., 2016; Collip et al., 2013; Ellett et al., 2008; Foster et al., 2010; Freeman et al., 2014; Freeman et al., 2015, 2016; Hepworth et al., 2011; Myers et al., 2011; Visceglia and Lewis, 2011). Yet, some of these studies are compromised by small sample size. Moreover, target populations (nonclinical individuals and patients with schizophrenia) and outcomes varied across studies. In addition, not all studies accord to predictions made by the model. For example, in a longitudinal study with adolescents/young adults, Sullivan et al. (2014) found that psychotic experiences but not depressive symptoms in 12-year-old children predicted psychotic experiences when they turned 18. In two other randomized controlled intervention trials that targeted depression, depressive but not positive symptoms were significantly reduced in schizophrenia patients (Moritz et al., 2015a, 2015b, 2016), which could, however, be due to the lack of a follow-up assessment. A brief compassion-focused imagery treatment improved emotional well-being in paranoid patients but did not impact paranoia (Ascone et al., 2017).

Some inconsistencies in findings likely mirror unresolved methodological problems. For example, standard regression procedures seem to overestimate the longitudinal association between depression and paranoia, even after adjusting for concurrent psychopathology because of the high cross-sectional association between symptoms of depression and paranoia measured at the same time-point, and the rather strong stability of the syndromes in terms of high auto-correlations across time. In this regard, cross-lagged models are deemed advantageous (for an overview, see Selig and Little, 2012).

In a recent study (Moritz et al., 2017b), we further explored the relationships between paranoia and depression. While we found that the affective symptoms emphasized in Freeman and Garety's model (e.g., worrying) predicted psychosis after six months and two years, the predictive strength of those depressive symptoms, which are not part of in their model, was comparable. No significant relationship emerged between depression and paranoia at baseline and six months. At trend, worry/rumination predicted paranoia from baseline to six months, which achieved significance from six months to two years. Avoidant behavior and sleep problems did not predict subsequent paranoia at any time point; rather, paranoia at baseline predicted poor sleep as well as overall depression severity at six months. However, as this study included neither a clinical nor a high-risk sample (in fact many participants were much older than the typical age at first episode), this finding does not refute the aforementioned causal model.

1.3. Psychosis, particularly paranoia, as a precursor or even risk factor for depression

While the majority of contemporary research is targeted at the possible predictive role depression plays for paranoia, the opposite relationship, for which the term "post-psychotic depression" has been coined, also deserves consideration. Early research has implicated side effects of antipsychotic treatment as a cause for subsequent depression (Floru et al., 1975), while more recent studies have shifted the perspective to psychological factors. Post-psychotic depression occurs once the "curtain of paranoia" is lifted; that is, once an individual realizes the consequences of his or her actions, which often range from shattered social relationships and isolation (Sandhu et al., 2013) to severe judicial problems in case of violent acts against people or objects. Birchwood, Upthegrove and others view the psychotic episode as a traumatic event that elicits feelings of loss (Sandhu et al., 2013), humiliation, shame (Turner et al., 2013; Upthegrove, 2009; Upthegrove et al., 2014) and perceived low social rank (Birchwood et al., 2005). These factors together with enhanced illness insight (Iqbal et al., 2000) are risk factors for depression and even suicide (Jackson et al., 2011; Schwartz-Stav et al., 2006).

\subsection{The present study}

To address some of the aforementioned limitations of prior research, particularly small sample sizes, recruitment of non-clinical samples and methodological problems associated with simple regression, in the present study we investigated a large patient sample currently being treated for a schizophrenia spectrum disorder (either in- or outpatients) at three different points in time (baseline, six weeks, six months postassessment). Analyses were conducted using cross-lagged models. Paranoia was assessed with the respective items of the Positive and Negative Syndrome Scale (PANSS) as well as the delusions subscale of the Psychotic Symptom Rating Scales (PSYRATS). Depressive symptoms were assessed with the PHQ-9 as well as the Calgary Depression Scale for Schizophrenia (CDSS). While we expected a direct path from depression to paranoia, we were also prepared to find the opposite effect as paranoia likely exerts a detrimental effect on well-being.

\section{Methods}

\subsection{Sample}

The sample was comprised of patients with a primary diagnosis of psychosis (including schizophrenia and schizoaffective disorder but not schizotypal personality disorder) as diagnosed by an experienced clinician based on the Mini International Neuropsychiatric Interview (M.I.N.I.) and other diagnostic information. Patients were between 18 and 65 years old and able to provide written informed consent. No symptom severity cutoff was applied. Patients were recruited from the in- and outpatient psychosis units of the University Medical Center Hamburg-Eppendorf, as well as through word of mouth, practitioners in the community and advertisements. The study was approved by the ethics board of the German Psychological Association (SM 102012_amd_032015) and registered in the German Clinical Trials Register (DRKS 00008001).

The following additional exclusion criteria were applied: acute suicidality/concrete suicidal plans; presence of severe neurological diseases (e.g., dementia, epilepsy); alcohol or drug dependence (alcohol or drug abuse were tolerated); IQ $<70$ points as measured with the "Worthschatztest" (Schmidt and Metzler, 1992); score $\geq 5$ for uncooperative behavior (item G8) and hostility (item P7) as measured by the Positive and Negative Syndrome Scale (PANSS); participation in MCT + in the past 12 months.

Most patients were chronic and had been admitted to a psychiatric hospital several times $(M=5.82)$. For only $15.6 \%$ of the sample the present stay was their first (see Table 1 ).

\subsection{Measures}

\subsubsection{Mini International Neuropsychiatric Interview (M.I.N.I.) 5.0 .0}

The M.I.N.I. Version 5.0.0 (Sheehan et al., 1998) is a structured interview for the diagnosis of axis I disorders according to DSM-IV. The 
Table 1

Demographic, treatment-related and psychopathological characteristics of the sample at baseline $(N=250)$.

\begin{tabular}{ll}
\hline Variables & $\begin{array}{l}\text { Mean (standard deviation) } \\
\text { or frequency }\end{array}$ \\
\hline Demographic variables & \\
Age in years & $39.28(11.08)$ \\
Gender (male, female) & $144 / 106(57.6 \%, 42.4 \%)$ \\
Education level (at least 12th grade vs. $<12$ years) & $126 / 124(50.5 \%, 49.5 \%)$ \\
Formal education in years & $11.09(1.92)$ \\
Post-secondary education in years & $3.77(3.00)$ \\
Treatment-related variables & \\
Admissions (including present one); \% first & $5.82(5.79) ; 15.6 \%$ first \\
admission & admission $(n=39)$ \\
Depression/Dysthymia (acute, acute and previously, & $31 / 36 / 79 / 104(12.4 \%$, \\
previously only, none) & $13.4 \%, 31.6 \%, 41.6 \%)$ \\
Inpatient/outpatient/semi-residential/none & $133 / 51 / 10 / 56(53.2 \%$, \\
& $20.4 \%, 4.0 \%, 22.4 \%)$ \\
Medication (antipsychotic agent, tranquilizer only, & $242 / 3 / 1 / 4$ \\
antidepressant(s) only, none) & $(96.8 \% / 1.2 \% / 0.4 \% / 1.6 \%)$ \\
Psychopathology & \\
PANSS delusion score & $10.18(4.30)$ \\
PSYRATS delusions score & $9.94(7.50)$ \\
PHQ-9 & $10.91(5.71)$ \\
CDSS & $5.10(4.28)$ \\
PANSS total score & $60.16(16.29)$ \\
\hline
\end{tabular}

Notes. CDSS = Calgary Depression Scale for Schizophrenia, PANSS = Positive and Negative Syndrome Scale (PANSS), PHQ-9 = Patient Health Questionnaire- 9 (depression), PSYRATS $=$ Psychosis Rating Scales, Delusions Subscale

sections for depression, dysthymia, suicidality, mania, substance use disorders, psychotic disorders, panic disorder, agoraphobia, social phobia, obsessive-compulsive disorder, post-traumatic stress disorder, generalized anxiety disorder and eating disorders were administered. The original scale was supplemented by self-devised items to ensure a diagnosis according to DSM-5.

\subsubsection{Positive and Negative Syndrome Scale (PANSS)}

The PANSS is a semi-structured interview that includes a total of 30 items capturing many symptoms typical of schizophrenia as well as more general psychopathology over the last seven days (Kay et al., 1987). These items are scored on a scale ranging from 1 to 7 , with 7 being the most severe rating. In addition, six items from the Positive and Negative and Disorganized Symptoms Scale (PANADSS) (Moritz et al., 2001) were added to this instrument, which specify PANSS items P2 (formal mental disorder), N1 (affect flattening), and P3 (hallucinations). For item P2, the PANADSS distinguishes between association loosening and speech blockage/mutism, for item N1, it distinguishes between flat and inadequate affect, and for item P3, it differentiates between acoustic and other hallucinations. For this study, the items P1 (Delusions), P5 (Delusions of Grandeur), P6 (Delusions of Mistrust/Persecution) and G9 (Unusual Thought Contents) were aggregated to obtain a paranoia score (range $4-28$ points).

\subsubsection{Psychosis Rating Scales (PSYRATS)}

The PSYRATS is an expert rating sale consisting of two subscales, which inquire about auditory hallucinations and delusions over the course of the last seven days (Haddock et al., 1999). Response options range from 0 to 4 resulting in a possible total sum value between 0 and 24 points. Hallucinations and delusions are captured in a more differentiated way than in the PANSS (e.g., loudness of voices, degree of conviction for false beliefs).

\subsubsection{Calgary Depression Scale for Schizophrenia (CDSS)}

The CDSS is a semi-structured expert rating scale which contains nine items and was specifically developed to measure depressive symptoms in schizophrenia beyond positive and negative symptoms (Addington et al., 1992). Construct validity has been confirmed by substantial correlations with other depression scales, such as the Hamilton Depression Rating Scale or the Beck Depression Inventory. The instrument has an excellent interrater reliability for the German version used with a $\kappa$ coefficient of 0.97 (Müller et al., 1999). All nine items are rated on a Likert scale from 0 to 3 and refer to the last seven days, where item 9 reflects the interviewer's observation. The total score ranges from 0 to 27 points.

\subsubsection{The Patient Health Questionnaire-9 (PHQ-9)}

The PHQ-9 (Gilbody et al., 2007; Kroenke et al., 2001) was used to assess subjective depressive symptoms. Its items match the nine diagnostic criteria for depression according to DSM-IV (scoring: not at all $(=0)$, several days $(=1)$, more than half the days $(=2)$, nearly every day (=3)). The scale was derived from the Primary Care Evaluation of Mental Disorders (PRIMEMD) and its psychometric properties are good (Gilbody et al., 2007).

\subsection{Statistical analyses}

All analyses were performed with Mplus version 8.0 (Muthén and Muthén, 2015). To test the measurement models for depression and paranoia at all assessment-points, we first computed confirmatory factor analyses (CFA). The latent variable "paranoia" was formed by two indicators: the summary score for paranoia items from the PANSS (items P1, P5, P6, G9) and PSYRATS (Haddock et al., 1999). The latent variable "depression" was also formed by two indicators: the total score of the CDSS (Addington et al., 1992) and the PHQ-9 (Gilbody et al., 2007; Kroenke et al., 2001). By using aggregated scores, we intended to increase the reliability of parameter estimates (Little et al., 2013). The longitudinal measurement invariance of the measurement models was investigated using a single group approach by first running unconstrained models with freely estimated parameter estimates. We then constrained factor loadings in tandem to be equal over time. The unconstrained and the constrained models were compared in terms of changes in Goodness of Fit and modification indices (Brown, 2015; Little, 2013). In Appendix A, we also report item-wise predictors. However, interpretation of these models is constrained by low reliability.

We used multiple imputations for all variables included in the crosslagged panel models while assuming random missingness. We employed the Bayes estimator (Asparouhov and Muthén, 2010), which employs Markov chain Monte Carlo (MCMC) algorithms, to impute missing data ( 100 imputed datasets were generated; results are reported based on pooled analyses of these datasets). Sensitivity analyses revealed similar result patterns for multiple imputations or complete cases. Model fit was determined by the following commonly used indices: Comparative Fit Index (CFI), Root Mean Square Error of Approximation (RMSEA) and Tucker Lewis Index (TLI).

Reciprocal effects among depressive and paranoid symptom across time were tested by calculating cross-lagged panel models, which provide estimates for the stability of constructs by regressing later measures onto earlier measures of the same construct to produce stability coefficients. Earlier measures of one construct (e.g., depression) are used to predict the later measure of the other construct (e.g., paranoia), which is called a cross-lagged association (Curran, 2000). Thereby, cross-lagged panel models allow for simultaneous estimation of reciprocal influences of depression and paranoia over and above the prior level of these constructs. We controlled our crosslagged panel models for age, gender and education. Furthermore, indicator-specific effects were estimated to model the shared method variance of indicators for both depression and paranoia over time instead of calculating correlated error variables to avoid an underestimation of reliability of indicators (Geiser and Lockhart, 2012). As model fit indices are limited in Mplus using Bayes estimator, we reported model fit based on Maximum Likelihood estimation. Effect sizes were expressed as correlation coefficients and pre-post effect sizes ([Mpost 
Means, standard deviations and changes over time in paranoia and depression.

\begin{tabular}{|c|c|c|c|c|c|c|c|c|c|c|c|c|}
\hline & \multicolumn{2}{|l|}{ T0 } & \multicolumn{2}{|l|}{$\mathrm{T} 1$} & \multicolumn{2}{|l|}{$\mathrm{T} 2$} & \multirow[t]{2}{*}{ Wald-test $\mathrm{T} 0 / \mathrm{T} 1^{\mathrm{a}}$} & \multirow[t]{2}{*}{$\mathrm{p}$} & \multirow{2}{*}{$\begin{array}{l}\text { T0-T1 } \\
\text { ES }\end{array}$} & \multirow[t]{2}{*}{ Wald-test $\mathrm{T} 1 / \mathrm{T} 2^{\mathrm{a}}$} & \multirow[t]{2}{*}{$\mathrm{p}$} & \multirow{2}{*}{$\begin{array}{l}\text { T0-T2 } \\
\text { ES }\end{array}$} \\
\hline & Mean & $(\mathrm{SD})$ & Mean & $(\mathrm{SD})$ & Mean & $(\mathrm{SD})$ & & & & & & \\
\hline Paranoia (PANSS) & 10.18 & $(4.29)$ & 8.09 & $(3.94)$ & 7.75 & $(4.08)$ & 32.08 & $<0.001$ & 0.49 & 36.94 & $<\mathbf{0 . 0 0 1}$ & 0.57 \\
\hline Paranoia (Psyrats) & 9.98 & $(7.50)$ & 6.90 & $(7.24)$ & 6.79 & $(7.34)$ & 23.75 & $<0.001$ & 0.41 & 22.53 & $<0.001$ & 0.43 \\
\hline Depression (PHQ) & 11.11 & $(7.78)$ & 9.13 & $(5.51)$ & 9.27 & $(5.52)$ & 15.24 & $<0.001$ & 0.25 & 9.57 & $<0.01$ & 0.24 \\
\hline PHQ 1: Anhedonia & 1.21 & $(0.97)$ & 1.05 & $(0.86)$ & 1.22 & $(0.90)$ & 3.31 & 0.069 & 0.16 & 0.02 & 0.886 & -0.01 \\
\hline PHQ 2: Depressed mood & 1.34 & $(0.93)$ & 1.11 & $(0.89)$ & 1.11 & $(0.87)$ & 7.54 & $<\mathbf{0 . 0 1}$ & 0.25 & 5.76 & 0.016 & 0.25 \\
\hline PHQ 3: Trouble sleeping & 1.57 & $(1.04)$ & 1.30 & (1.03) & 1.44 & $(1.06)$ & 8.59 & $<0.01$ & 0.26 & 1.46 & 0.227 & 0.13 \\
\hline PHQ 4: Feeling tired & 1.52 & $(1.01)$ & 1.36 & $(0.95)$ & 1.38 & $(0.95)$ & 4.20 & $<\mathbf{0 . 0 5}$ & 0.16 & 1.96 & 0.161 & 0.14 \\
\hline PHQ 5: Change in appetite & 1.21 & $(1.04)$ & 1.02 & $(0.98)$ & 0.98 & $(0.98)$ & 4.10 & $<0.05$ & 0.18 & 4.19 & $<0.05$ & 0.22 \\
\hline PHQ 6: Guilt, worthlessness & 1.26 & $(1.06)$ & 0.93 & $(0.91)$ & 0.96 & $(0.97)$ & 14.39 & $<0.001$ & 0.31 & 8.13 & $<0.01$ & 0.28 \\
\hline PHQ 7: Concentrating & 1.43 & $(1.00)$ & 1.12 & $(0.93)$ & 1.02 & $(0.91)$ & 12.67 & $<0.001$ & 0.31 & 20.14 & $<0.001$ & 0.41 \\
\hline PHQ 8: Slowed down/restless & 0.96 & $(0.98)$ & 0.70 & $(0.88)$ & 0.67 & $(0.85)$ & 10.84 & $<0.01$ & 0.27 & 9.73 & $<0.01$ & 0.30 \\
\hline PHQ 9: Suicidal thoughts & 0.58 & $(0.84)$ & 0.51 & $(0.82)$ & 0.43 & $(0.75)$ & 0.66 & 0.415 & 0.08 & 2.15 & 0.142 & 0.18 \\
\hline Depression (CDSS) & 5.09 & $(4.27)$ & 4.39 & $(4.04)$ & 4.29 & $(4.20)$ & 5.26 & $<0.05$ & 0.16 & 4.55 & $<0.05$ & 0.19 \\
\hline CDSS 1: Depression & 0.96 & $(0.97)$ & 0.91 & $(0.93)$ & 0.79 & $(0.87)$ & 0.58 & 0.448 & 0.05 & 4.59 & $<0.05$ & 0.18 \\
\hline CDSS 2: Hopelessness & 0.72 & $(0.83)$ & 0.62 & $(0.76)$ & 0.63 & $(0.79)$ & 2.17 & 0.141 & 0.12 & 1.86 & 0.173 & 0.11 \\
\hline CDSS 3: Self-depreciation & 0.78 & $(0.95)$ & 0.59 & $(0.83)$ & 0.65 & $(0.84)$ & 5.30 & $<0.05$ & 0.20 & 1.77 & 0.184 & 0.14 \\
\hline CDSS 4: Guilty ideas of reference & 0.41 & $(0.79)$ & 0.25 & $(0.54)$ & 0.30 & $(0.60)$ & 7.28 & $<\mathbf{0 . 0 1}$ & 0.20 & 2.83 & 0.093 & 0.14 \\
\hline CDSS 5: Pathological guilt & 0.48 & $(0.74)$ & 0.35 & $(0.65)$ & 0.34 & $(0.67)$ & 5.85 & $<0.05$ & 0.18 & 3.51 & 0.061 & 0.19 \\
\hline CDSS 6: Morning depression & 0.51 & $(0.74)$ & 0.54 & $(0.81)$ & 0.54 & $(0.77)$ & 0.15 & 0.702 & -0.04 & 0.05 & 0.829 & -0.04 \\
\hline CDSS 7: Early awakening & 0.50 & $(0.90)$ & 0.39 & $(0.82)$ & 0.36 & $(0.87)$ & 2.29 & 0.130 & 0.12 & 2.42 & 0.120 & 0.16 \\
\hline CDSS 8: Suicide & 0.18 & $(0.42)$ & 0.16 & $(0.42)$ & 0.15 & $(0.36)$ & 0.51 & 0.478 & 0.05 & 0.74 & 0.390 & 0.07 \\
\hline CDSS 9: Observed depression & 0.56 & $(0.75)$ & 0.50 & $(0.68)$ & 0.50 & $(0.68)$ & 1.19 & 0.276 & 0.08 & 1.16 & 0.282 & 0.08 \\
\hline
\end{tabular}

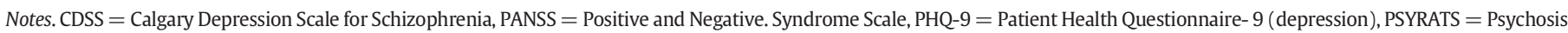
Rating Scales, Delusions Subscale.

- Mpre]/SDpre) for the descriptive analyses, and standardized partial regression coefficients for the cross-lagged panel models.

\section{Results}

\subsection{Descriptive statistics}

Data from 250 patients could be included at baseline. At T1, data from 205 participants (82.0\%) was available. Six months later (T2), approximately three-quarters of participants could be tested $(n=185$ patients; 74.0\%). Participants who did not take part in T1 had higher baseline depression scores on the PHQ $(p=0.03)$ than those who completed T1. No differences with regard to age, gender, education or depressive or paranoid symptoms were detected between completers and non-completers at T2. Table 1 summarizes the main demographic, psychopathological and treatment-related (e.g., medication) data.

At baseline, the modal patient was male in his late 30s. Half of the participants had 12 years or more education $(n=126,50.5 \%)$. Most participants were currently in psychiatric treatment, either as inpatients ( $n$ $=133,53.2 \%$ ) or outpatients $(n=51,20.4 \%$ ). All patients met criteria for a schizophrenia spectrum disorder. The majority of patients fulfilled criteria of a lifetime diagnosis of depression or dysthymia according to the M.I.N.I. - only $41.6 \%$ never met criteria for such diagnoses. The overall symptom severity of the PANSS was 60.2, which equates to rather mild symptomatology.

Table 2 presents descriptive statistics for paranoia and depressive symptoms over time, as well as results of $t$-tests for paired samples and effect sizes based on imputed data. Paranoia significantly decreased to a moderate degree between baseline and T1 as well as between T1 and T2. Decrease in depression was smaller than that for paranoia, but was significant at both assessment-points ( $\mathrm{T} 1$ and $\mathrm{T} 2$ ), especially with regard to depressed mood, change in appetite, feelings of guilt/worthlessness, concentration and being slowed down/restless.

\subsection{Measurement models and measurement invariance}

All measurement models revealed good model fit for paranoia (CFI $=1.00, \mathrm{TLI}=1.00, \mathrm{RMSEA}=0.00)$ and depression $(\mathrm{CFI}=1.00$, TLI $=1.01$, RMSEA $=0.00$ ) at each assessment-point. Measurement invariance was tested by comparing constrained and unconstrained models across time. Differences for model fit were CFI $=0.000$, TLI $=$ -0.002 , RMSEA $=0.006$ for paranoia and $\mathrm{CFI}=0.000$, TLI $=0.009$, RMSEA $=0.000$ for depression, which is acceptable (Chen, 2007; Cheung and Rensvold, 2002). Modification indices indicated adequate fit for the constrained models. This ensured that assessments measured the same constructs over time.

\subsection{Correlations between depressive and paranoid symptoms over time}

All model variables were significantly and positively associated with each other at all assessment points. The only exceptions were the nonsignificant correlations between both depression measures (i.e., CDSS and PHQ) at baseline and paranoia as measured by the PANSS at T1 and $\mathrm{T} 2$. In general, correlations were highest for measures at the same assessment-points.

\subsection{Stability over time}

In the cross-lagged panel model, regression coefficients of 0.88 (T0T1) and 0.86 (T1-T2) for paranoia indicate that levels of paranoia were stable over time paralleled by significant improvement across the three points of assessment. In comparison, stability of depression was modest (T0-T1: $\beta=0.46$; T1-T2: $\beta=0.52$ ) (see Fig. 1 ). Table 3 shows the stability coefficients using individual assessments instead of forming latent variables. Self-reported depression (PHQ-9) demonstrated higher stability from T0 to T1 than the expert-rated CDSS (similar values from T1 to T2), whereas for paranoia, the PANSS was more stable than the PSYRATS.

\subsection{Cross-lagged associations between depressive and paranoid symptoms over time}

Higher levels of paranoia at baseline were significantly associated with more depression at both T1 and at T2 (see Fig. 1). Depression at baseline predicted paranoia at $\mathrm{T} 1$, but no such association occurred for T2. Higher levels of depression at baseline were significantly associated with less paranoia at T1. With regard to covariates, age but not gender or years of education were significantly associated with paranoia such 
that older age was associated with more paranoia. None of the covariates showed any significant association with depression.

The individual standardized cross-lagged coefficients are shown in Appendix A. These parameter estimates are not as reliable as the full model with latent factors and should be interpreted with caution. As can be seen in Appendix A, six out of nine items from the CDSS could predict paranoia at T1 but only two (and with lesser weight) at T2. Items from the PHQ-9 were weak predictors overall.

\section{Discussion}

The present study aimed to shed further light on the temporal relationships between depressive symptoms and paranoia. A direct path was found from paranoia to depression at both post (6 weeks) and follow-up ( 6 months) assessments. The reverse path was only significant for post-assessment and was in the opposite direction than expected.

Our findings contribute to a growing literature demonstrating the psychological problems and hardships paranoia and other positive symptoms inflict on patients in the long run (see introduction). Clearly, our study does not rule out a path leading from depressive symptoms to paranoia as implicated in Freeman and Garety's model (Freeman, 2016; Freeman and Garety, 2014; Garety and Freeman, 2013) but such a linkage is perhaps more prominent in the other phases of the disorder (e.g., clinical high risk state, before relapse).

The present study has a number of strengths but also weaknesses. A strength is seen in the large sample of patients with a diagnosed schizophrenia spectrum disorder and the good retention rate. Moreover, the instruments are well established for the measurement of both paranoia and depression; test-retest reliability was satisfactory to good, particularly for paranoia. Importantly, we used a self-report as well as a clinician-rated instrument to assess depression as both sources obtain unique information (Uher et al., 2012). A serious limitation is that we have no data on longer intervals; results from midterm and long-term follow-ups may vary greatly (Moritz et al., 2017a, 2017b). Thus, we cannot say whether the relationship from paranoia towards depression is strengthened over time or even reverts. A problem with this and most other studies in the field is that no gold standard exists for the measurement of depression in schizophrenia. Findings tentatively suggest that results are not congruent for self-report and expert rating scales (Uher et al., 2012). Of more concern, a lot of different aspects (e.g., worry,
Table 3

Standardized stability coefficients over time for paranoia and depression.

\begin{tabular}{|c|c|c|c|c|c|c|}
\hline & \multicolumn{3}{|l|}{$\mathrm{T} 0 \rightarrow \mathrm{T} 1$} & \multicolumn{3}{|l|}{$\mathrm{T} 1 \rightarrow \mathrm{T} 2$} \\
\hline & beta & $95 \% \mathrm{CI}$ & posterior SD & beta & $95 \% \mathrm{CI}$ & Posterior SD \\
\hline \multicolumn{7}{|l|}{ Paranoia } \\
\hline PANSS & $0.49^{* * * *}$ & $0.40 ; 0.59$ & 0.04 & $0.71^{* * *}$ & $0.64 ; 0.78$ & 0.04 \\
\hline PSYRATS & $0.31^{* * *}$ & $0.20 ; 0.43$ & 0.06 & $0.57^{* * *}$ & $0.44 ; 0.67$ & 0.06 \\
\hline \multicolumn{7}{|c|}{ Depression } \\
\hline CDSS & $0.28^{* * *}$ & $0.18 ; 0.40$ & 0.06 & $0.42^{* * *}$ & $0.30 ; 0.52$ & 0.06 \\
\hline PHQ & $0.43^{* * *}$ & $0.35 ; 0.52$ & 0.04 & $0.46^{* * *}$ & $0.35 ; 0.55$ & 0.05 \\
\hline
\end{tabular}

Note. ${ }^{* * *} p<0.001$; no indicator-specific effect was estimated in these models as manifest individual assessments were used for paranoia and depression.

CDSS $=$ Calgary Depression Scale for Schizophrenia, PANSS = Positive and Negative Syndrome Scale (PANSS), PHQ-9 = Patient Health Questionnaire- 9 (depression), PSYRATS = Psychosis Rating Scales, Delusions Subscale.

rumination, explicit or implicit self-esteem, depression, etc.) captured by very different scales are subsumed under the umbrella term "negative affect". Studies in the field are therefore perhaps more inconsistent than it appears from abstracts. Moreover, although cross-lagged models are now regarded as one of the most appropriate statistical techniques to study relationships between model variables prospectively, they have also been subject to criticism (Hamaker et al., 2015). Finally, our sample was mainly chronic; few patients (15.6\%) were experiencing their first episode, which may have obscured stronger causal relationships between depression and paranoia as highlighted in the model by Freeman and Garety (see below).

Whatever the relationship between depression and paranoia across different stages of the schizophrenia spectrum disorder (e.g., paranoia predicting depression in remission, depression predicting paranoia in the clinical high risk state), we need to redefine the role of depression for the treatment of patients. So far, positive and negative symptoms of schizophrenia have been the primary treatment targets and typical outcomes of most contemporary research, which may partly be due to the fact that these symptoms are (allegedly) easier to treat with antipsychotic medication. In addition, positive and negative symptoms have represented hallmark symptoms of schizophrenia for decades, whereas depression has received attention only until recently. However, prevalence of depression in this population is high (Buckley et al., 2009) and patients themselves attribute a high priority to the

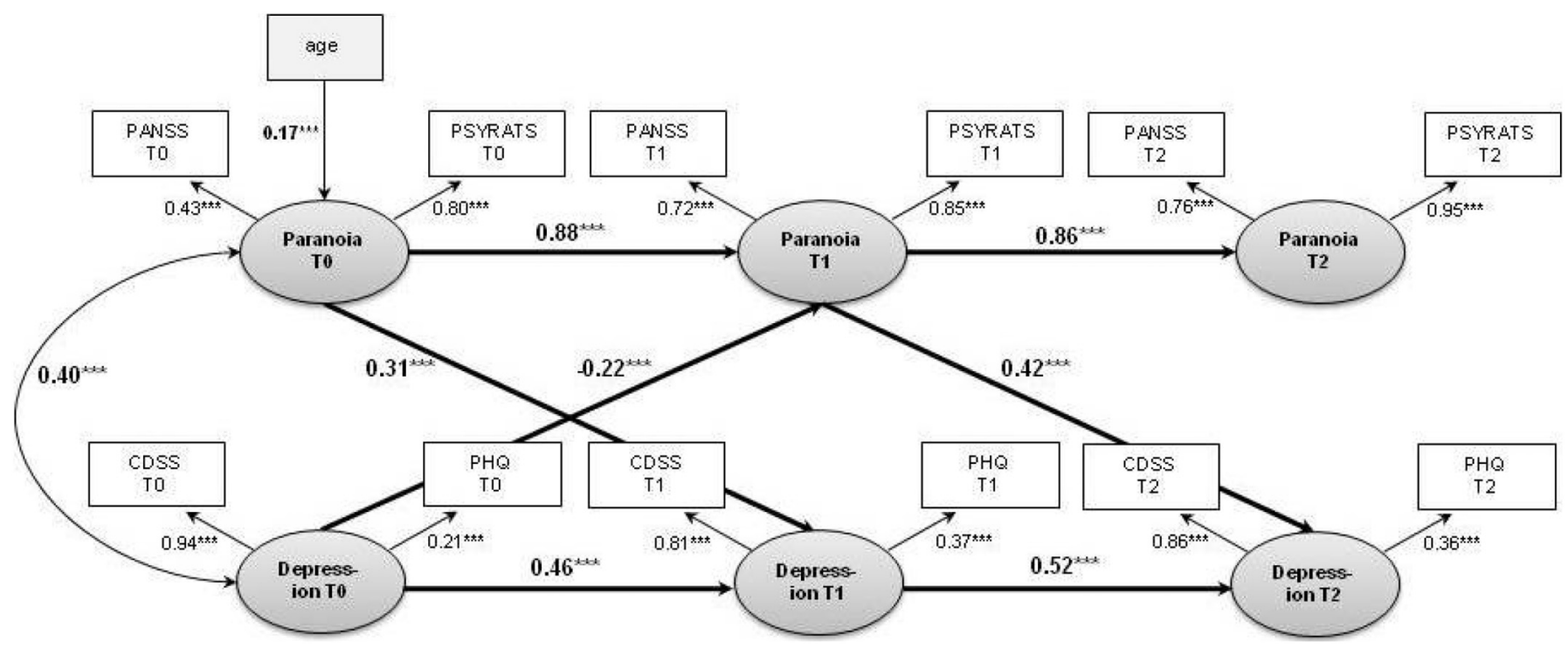

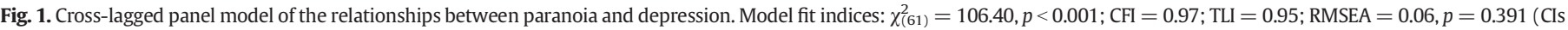

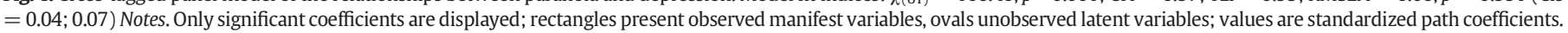
${ }^{* *} p<0.01,{ }^{* * *} p<0.001$. 
treatment of depressive symptoms (Byrne et al., 2010; Kuhnigk et al., 2012; Moritz et al., 2017a, 2017b). Depression is also related to suicidal ideation, self-stigma and treatment fatalism.

Our results corroborate prior studies showing that patients with paranoia may need special care in terms of both psychosis and depression prophylaxis after discharge. This holds true especially for those whose social network has been strained by psychosis (e.g., in case they insulted, attacked or neglected other people, especially significant others during psychosis), which may cause despair and shame (Sandhu et al., 2013). Losing one's job or participating only in low-paid work in sheltered employment may contribute to feelings of low social rank (Birchwood et al., 2005), which heightens the risk for depression.

Like in our forerunner study with nonclinical participants, depressive and paranoid symptoms were stable over time. At baseline, paranoia was highly correlated with depression confirming that affective and psychotic symptoms are more closely associated than assumed from a "Kraepelian" viewpoint.

Although our findings do not support a causal association between high depressive symptoms and subsequent paranoia (at least at the clinical stages tested in our sample), these associations are worth further investigation; affective symptoms are associated with suffering and thus remain an important clinical target. The unexpected negative association between baseline depression and post paranoia needs to be replicated and should be regarded an outlier until then. Most studies found either null or positive associations and we would like to reiterate our former call not only to examine the statistical associations between psychotic and affective symptoms, but to truly understand the dynamics behind them (Westermann et al., 2015). Such linkages may be quite idiosyncratic and thus may only hold for specific subgroups. To illustrate, poor drive, avoidance and withdrawal limit corrective experiences and positive feedback from others. As Zimbardo (1999) has argued, this likely fosters a sentiment of abandonment, which may result in paranoia. For a subgroup of patients, poor self-esteem may trigger the wish for personal importance, which could act as a maintenance factor for delusional ideas that contain grandiose elements (Moritz et al., 2015a, 2015b; Sundag et al., 2015). Sleep problems and cognitive problems due to depressive thoughts can interfere with cognition and prompt "weird ideas" (Moritz et al., 2017a, 2017b). Put differently, we should try to track the domino pieces that may go from depression to paranoia.

It deserves to be tested whether it is the social and functional consequences of negative affect that contribute to paranoia and not the depressive symptoms as such. As argued above, this may also apply for the opposite relationship: paranoia likely interferes with the social and work environment and any such challenges may prompt depressive symptoms via feelings of shame, entrapment and a reduction in perceived social rank. Taken together, we agree with Freeman et al. (2014) that improving emotional well-being including depression is important in its own right whether or not it is related to future positive symptoms such as paranoia.

Supplementary data to this article can be found online at https://doi. org/10.1016/j.schres.2019.06.022.

\section{Contributors}

Steffen Moritz, Ruth Veckenstedt and Lea-Elena Braunschneider developed the research idea. Brooke Schneider was PI of the study. Alisa Manske and Stefanie J Schmidt performed the cross-lagged analyses. Thies Lüdtke was especially involved in preparing the introduction and discussion. All authors have written and proofread the manuscript.

\section{Role of funding source}

This study was supported by a grant from the German Research Foundation (Deutsche Forschungsgemeinschaft) to Professor Moritz (MO 969/16-1).

\section{Declaration of Competing Interest}

None.

\section{Acknowledgement}

We thank Katharina Rauprich, Jana Ahlf-Schumacher, Milena Rahmede and Saskia Hanft-Robert for help with recruitment.

\section{References}

Addington, D., Addington, J., Maticka-Tyndale, E., \& Joyce, J. (1992). Reliability and validity of a depression rating scale for schizophrenics. Schizophr. Res., 6(3), 201-208. https://doi.org/10.1016/0920-9964(92)90003-N.

Ascone, L., Sundag, J., Schlier, B., Lincoln, T.M., 2017. Feasibility and effects of a brief compassion-focused imagery intervention in psychotic patients with paranoid ideation: a randomized experimental pilot study. Clin. Psychol. Psychother. 24 (2), 348-358. https://doi.org/10.1002/cpp.2003.

Asparouhov, T., Muthén, B., 2010. Bayesian analysis of latent variable models using Mplus. Retrieved April, 14, 2016 from. http://www.statmodel.com/download/ BayesAdvantages 18.pdf.

Ben-Zeev, D., Ellington, K., Swendsen, J., Granholm, E., 2011. Examining a cognitive model of persecutory ideation in the daily life of people with schizophrenia: a computerized experience sampling study. Schizophr. Bull. 37 (6), 1248-1256. https://doi.org/ $10.1093 /$ schbul/sbq041.

Birchwood, M., Iqbal, Z., Upthegrove, R., 2005. Psychological pathways to depression in schizophrenia. Eur. Arch. Psychiatry Clin. Neurosci. 255 (3), 202-212. https://doi org/10.1007/s00406-005-0588-4.

Brown, T.A., 2015. Confirmatory Factor Analysis for Applied Research. second edition. Guilford Press, New York, N.Y.

Buckley, P.F., Miller, B.J., Lehrer, D.S., Castle, D.J., 2009. Psychiatric comorbidities and schizophrenia. Schizophr. Bull. 35 (2), 383-402. https://doi.org/10.1093/schbul/ sbn135.

Bullock, G., Newman-Taylor, K., Stopa, L., 2016. The role of mental imagery in non-clinical paranoia. J. Behav. Ther. Exp. Psychiatry 50, 264-268. https://doi.org/10.1016/j. jbtep.2015.10.002.

Byrne, R., Davies, L., Morrison, A.P., 2010. Priorities and preferences for the outcomes of treatment of psychosis: a service user perspective. Psychosis 2 (3), 210-217. https://doi.org/10.1080/17522430903456913.

Cardno, A.G., Owen, M.J., 2014. Genetic relationships between schizophrenia, bipolar disorder, and schizoaffective disorder. Schizophr. Bull. 40 (3), 504-515. https://doi.org/ $10.1093 /$ schbul/sbu016.

Chen, F.F., 2007. Sensitivity of goodness of fit indexes to lack of measurement invariance. Struct. Equ. Model. 14 (3), 464-504.

Cheung, G.W., Rensvold, R.B., 2002. Evaluating goodness-of-git indexes for testing measurement invariance. Struct. Equ. Model. Multidiscip. J. 9 (2), 233-255. https://doi. org/10.1207/S15328007SEM0902_5.

Collip, D., Geschwind, N., Peeters, F., Myin-Germeys, I., van Os, J., Wichers, M., 2013. Putting a hold on the downward spiral of paranoia in the social world: a randomized controlled trial of mindfulness-based cognitive therapy in individuals with a history of depression. PLoS One 8 (6), e66747. https://doi.org/10.1371/journal. pone.0066747.

Crow, T. J. (1995). A continuum of psychosis, one human gene, and not much else-the case for homogeneity. Schizophr. Res., 17(2), 135-145. https://doi.org/10.1016/ 0920-9964(95)00059-U.

Curran, P.J., 2000. A latent curve framework for the study of developmental trajectories in adolescent substance use. In: Rose, J., Chassin, L., Presson, C., Sherman, J. (Eds.), Multivariate Applications in Substance Use Research. New Methods for New Questions. Erlbaum, Hillsdale, NJ , pp. 1-42 Retrieved from. http://search.epnet.com/login. aspx direct $=$ true $\& \mathrm{db}=$ psyh\&an $=2000-07400-001$.

Ellett, L., Freeman, D., Garety, P.A., 2008. The psychological effect of an urban environment on individuals with persecutory delusions: the Camberwell walk study. Schizophr Res. 99 (1-3), 77-84. https://doi.org/10.1016/j.schres.2007.10.027.

Floru, L., Heinrich, K., Wittek, F., 1975. The problem of post-psychotic schizophrenic depressions and their pharmacological induction. Int. Pharmacopsychiatry 10 (4), 230-239. https://doi.org/10.1159/000468199.

Foster, C., Startup, H., Potts, L., Freeman, D., 2010. A randomised controlled trial of a worry intervention for individuals with persistent persecutory delusions. J. Behav. Ther. Exp. Psychiatry 41 (1), 45-51. https://doi.org/10.1016/j.jbtep.2009.09.001.

Freeman, D. (2016). Persecutory delusions: a cognitive perspective on understanding and treatment. Lancet Psychiatry, 3(7), 685-692. https://doi.org/10.1016/S2215-0366 (16)00066-3.

Freeman, D., Garety, P., 2014. Advances in understanding and treating persecutory delusions: a review. Soc. Psychiatry Psychiatr. Epidemiol. 49 (8), 1179-1189. https:// doi.org/10.1007/s00127-014-0928-7.

Freeman, D., Pugh, K., Dunn, G., Evans, N., Sheaves, B., Waite, F., Fowler, D., 2014. An early phase II randomised controlled trial testing the effect on persecutory delusions of using CBT to reduce negative cognitions about the self: the potential benefits of enhancing self confidence. Schizophr. Res. 160 (1-3), 186-192. https://doi.org/ 10.1016/j.schres.2014.10.038.

Freeman, D. Dunn, G., Startup, H. Pugh, K Cordwell J, Mander, H. Kingdon, D. (2015). Effects of cognitive behaviour therapy for worry on persecutory delusions in patients with psychosis (WIT): a parallel, single-blind, randomised controlled trial with a mediation analysis. Lancet Psychiatry, 2(4), 305-313. https://doi.org/10.1016/S22150366(15)00039-5.

Freeman, D., Bradley, J., Waite, F., Sheaves, B., DeWeever, N., Bourke, E., Dunn, G., 2016. Targeting recovery in persistent persecutory delusions: a proof of principle study of a new translational psychological treatment (the feeling safe programme). Behav. Cogn. Psychother. 44 (5), 539-552. https://doi.org/10.1017/S1352465816000060.

Garety, P.A., Freeman, D., 2013. The past and future of delusions research: from the inexplicable to the treatable. Br. J. Psychiatry 203 (5), 327-333. https://doi.org/10.1192/ bjp.bp. 113.126953.

Geiser, C., Lockhart, G., 2012. A comparison of four approaches to account for method effects in latent state-trait analyses. Psychol. Methods 17 (2), 255-283. https://doi.org/ 10.1037/a0026977. 
Gilbody, S., Richards, D., Brealey, S., Hewitt, C., 2007. Screening for depression in medica settings with the Patient Health Questionnaire (PHQ): a diagnostic meta-analysis. J. Gen. Intern. Med. 22 (11), 1596-1602. https://doi.org/10.1007/s11606-007-0333-y.

Haddock, G., McCarron, J., Tarrier, N., Faragher, E.B., 1999. Scales to measure dimensions of hallucinations and delusions: the psychotic symptom rating scales (PSYRATS). Psychol. Med. 29 (4), 879-889. https://doi.org/10.1017/S0033291799008661.

Häfner, H., Maurer, K., An Der Heiden, W., 2013. ABC schizophrenia study: An overview of results since 1996. Soc. Psychiatry Psychiatr. Epidemiol. 48 (7), 1021-1031. https:// doi.org/10.1007/s00127-013-0700-4.

Hamaker, E. L., Kuiper, R. M., \& Grasman, R. P. (2015). A critique of the cross-lagged pane model. Psychol. Methods, 20(1), 102-116. http://doi.org/https://doi.org/10.1037/ a0038889.

Hartley, S., Haddock, G., Vasconcelos e Sa, D., Emsley, R., Barrowclough, C., 2014. An experience sampling study of worry and rumination in psychosis. Psychol. Med. 44 (8), 1605-1614. https://doi.org/10.1017/S0033291713002080.

Hepworth, C., Startup, H., Freeman, D., 2011. Developing treatments of persistent persecutory delusions. J. Nerv. Ment. Dis. 199 (9), 653-658. https://doi.org/10.1097/ NMD.0b013e318229cfa8.

Iqbal, Z., Birchwood, M., Chadwick, P., Trower, P., 2000. Cognitive approach to depression and suicidal thinking in psychosis. Br. J. Psychiatry 177 (6), 522-528. https://doi.org/ 10.1192/bjp.177.6.522.

Jackson, C., Bernard, M., Birchwood, M., 2011. The efficacy of psychotherapy in reducing post-psychotic trauma. Epidemiol. Psychiatr. Sci. 20 (2), 127-131. https://doi.org/ 10.1017/S2045796011000205.

Kay, S.R., Fiszbein, A., Opler, L.A., 1987. The Positive and Negative Syndrome Scale (PANSS) for schizophrenia. Schizophr. Bull. 13 (2), 261-276. https://doi.org/ 10.1093/schbul/13.2.261.

Kramer, I., Simons, C.J.P., Wigman, J.T., Collip, D., Jacobs, N., Derom, C., Wichers, M., 2014 Time-lagged moment-to-moment interplay between negative affect and paranoia: new insights in the affective pathway to psychosis. Schizophr. Bull. 40 (2) 278-286. https://doi.org/10.1093/schbul/sbs194.

Kroenke, K., Spitzer, R.L., Williams, J.B.W., 2001. The PHQ-9: validity of a brief depression severity measure. J. Gen. Intern. Med. 16 (9), 606-613. https://doi.org/10.1046/ j.1525-1497.2001.016009606.x.

Kuhnigk, O., Slawik, L., Meyer, J., Naber, D., Reimer, J., 2012. Valuation and attainment of treatment goals in schizophrenia: perspectives of patients, relatives, physicians, and payers. J. Psychiatr. Pract. 18 (5), 321-328. https://doi.org/10.1097/01. pra.0000419816.75752.65.

Little, T.D., 2013. Longitudinal Structural Equation Modeling. New York. Guilford Press, New York, NY.

Little, T.D., Rhemtulla, M., Gibson, K., Schoemann, A.M., 2013. Why the items versus parcels controversy needn't be one. Psychol. Methods 18 (3), 285-300. https://doi.org/ $10.1037 / \mathrm{a} 0033266$.

Lüdtke, T., Kriston, L., Schröder, J., Lincoln, T.M., Moritz, S., 2017. Negative affect and a fluctuating jumping to conclusions bias predict subsequent paranoia in daily life: an online experience sampling study. J. Behav. Ther. Exp. Psychiatry 56, 106-112. https:// doi.org/10.1016/j.jbtep.2016.08.014

Moritz, S., Andresen, B., Jacobsen, D., Mersmann, K., Wilke, U., Lambert, M., ... Krausz, M. (2001). Neuropsychological correlates of schizophrenic syndromes in patients treated with atypical neuroleptics. Eur. Psychiatry, 16(6), 354-361. https://doi.org/ 10.1016/S0924-9338(01)00591-0.

Moritz, S., Cludius, B., Hottenrott, B., Schneider, B.C., Saathoff, K., Kuelz, A.K., Gallinat, J. 2015a. Mindfulness and relaxation treatment reduce depressive symptoms in individuals with psychosis. Eur. Psychiatry 30 (6), 709-714. https://doi.org/10.1016/j. eurpsy.2015.05.002

Moritz, S., Rietschel, L., Veckenstedt, R., Bohn, F., Schneider, B.C., Lincoln, T.M., Karow, A 2015b. The other side of "madness": frequencies of positive and ambivalent attitudes towards prominent positive symptoms in psychosis. Psychosis 7 (1), 14-24. https:// doi.org/10.1080/17522439.2013.865137.

Moritz, S., Schröder, J., Klein, J.P., Lincoln, T.M., Andreou, C., Fischer, A., Arlt, S., 2016. Effects of online intervention for depression on mood and positive symptoms in schizophrenia. Schizophr. Res. 175 (1-3), 216-222. https://doi.org/10.1016/j.schres.2016.04.033.

Moritz, S., Berna, F., Jaeger, S., Westermann, S., Nagel, M., 2017a. The customer is always right? Subjective target symptoms and treatment preferences in patients with psychosis. Eur. Arch. Psychiatry Clin. Neurosci. 267 (4), 335-339. https://doi.org/ 10.1007/s00406-016-0694-5.

Moritz, S., Göritz, A.S., McLean, B., Westermann, S., Brodbeck, J., 2017b. Do depressive symptoms predict paranoia or vice versa? J. Behav. Ther. Exp. Psychiatry 56 113-121. https://doi.org/10.1016/j.jbtep.2016.10.002.

Moritz, S., Engel, M., Fritzsche, A., Meiseberg, J., Klingberg, S., Hesse, K., 2019. A plea for a transdiagnostic conceptualization of negative symptoms and for consistent psychiatric vocabulary. Schizophr. Res.
Müller, M. J., Marx-Dannigkeit, P., Schlösser, R., Wetzel, H., Addington, D., \& Benkert, O. (1999). The Calgary depression rating scale for schizophrenia: development and interrater reliability of a German version (CDSS-G). J. Psychiatr. Res., 33(5), 433-443. https://doi.org/10.1016/S0022-3956(99)00018-7.

Muthén, L., Muthén, B., 2015. Mplus User's guide. Seventh edition. Muthén \& Muthén, Los Angeles, CA.

Myers, E., Startup, H., Freeman, D., 2011. Cognitive behavioural treatment of insomnia in individuals with persistent persecutory delusions: a pilot trial. J. Behav. Ther. Exp. Psychiatry 42 (3), 330-336. https://doi.org/10.1016/j.jbtep.2011.02.004.

Myin-Germeys, I., Kasanova, Z., Vaessen, T., Vachon, H., Kirtley, O., Viechtbauer, W., Reininghaus, U., 2018. Experience sampling methodology in mental health research: new insights and technical developments. World Psychiatry 17 (2), 123-132. https:// doi.org/10.1002/wps.20513.

Sandhu, A., Ives, J., Birchwood, M., Upthegrove, R., 2013. The subjective experience and phenomenology of depression following first episode psychosis: a qualitative study using photo-elicitation. J. Affect. Disord. 149 (1-3), 166-174. https://doi.org/ 10.1016/j.jad.2013.01.018.

Schmidt, K.H., Metzler, P., 1992. Wortschatztest [Vocabulary Test]. Beltz, Weinheim.

Schwartz-Stav, O., Apter, A., Zalsman, G., 2006. Depression, suicidal behavior and insight in adolescents with schizophrenia. Eur. Child Adolesc. Psychiatry 15 (6), 352-359. https://doi.org/10.1007/s00787-006-0541-8.

Selig. J.P., Little, T.D., 2012. Autoregressive and cross-lagged panel analysis for longitudinal data. In: Laursen, B., Little, T.D., Card, N.A. (Eds.), Handbook of Developmental Research Methods. The Guilford Press, New York, N.Y, pp. 265-288.

Sheehan, D.V., Lecrubier, Y., Sheehan, K.H., Amorim, P., Janavs, J., Weiller, E., ... Dunbar, G., 1998. The MINI international neuropsychiatric interview (M.I.N.I.): the development and validation of a structured diagnostic psychiatric interview. J. Clin. Psychiatry 59 (Suppl. 20), 22-33.

So, S.H.-W Chau, A.K.C. Peters, E.R., Swendsen, J, Garety, P.A., Kapur, S. 2018. Momentto-moment associations between negative affect, aberrant salience, and paranoia. Cogn. Neuropsychiatry 23 (5), 299-306. https://doi.org/10.1080/ 13546805.2018.1503080.

Sullivan, S.A., Wiles, N., Kounali, D., Lewis, G., Heron, J., Cannon, M., Zammit, S., 2014. Longitudinal associations between adolescent psychotic experiences and depressive symptoms. PLoS One 9 (8), e105758. https://doi.org/10.1371/journal.pone.0105758.

Sundag J. Lincoln, T.M. Hartmann, M.M. Moritz, S, 2015. Is the content of persecutory delusions relevant to self-esteem? Psychosis 7 (3), 237-248. https://doi.org/ 10.1080/17522439.2014.947616

Turner, M.H., Bernard, M., Birchwood, M., Jackson, C., Jones, C., 2013. The contribution of shame to post-psychotic trauma. Br. J. Clin. Psychol. 52 (2), 162-182. https://doi.org/ 10.1111/bjc.12007.

Udachina, A., Varese, F., Myin-Germeys, I., Bentall, R.P., 2014. The role of experiential avoidance in paranoid delusions: an experience sampling study. Br. J. Clin. Psychol. 53 (4), 422-432. https://doi.org/10.1111/bjc.12054.

Uher, R., Perlis, R.H., Placentino, A., Dernovšek, M.Z., Henigsberg, N., Mors, O., Farmer, A., 2012. Self-report and clinician-rated measures of depression severity: can one replace the other? Depress. Anxiety 29 (12), 1043-1049. https://doi.org/10.1002/ da. 21993.

Upthegrove, R., 2009. Depression in schizophrenia and early psychosis: implications for assessment and treatment. Adv. Psychiatr. Treat. 15 (5), 372-379. https://doi.org/ 10.1192/apt.bp.108.005629.

Upthegrove, R., Ross, K., Brunet, K., McCollum, R., Jones, L., 2014. Depression in first episode psychosis: the role of subordination and shame. Psychiatry Res. 217 (3), 177-184. https://doi.org/10.1016/j.psychres.2014.03.023.

Visceglia, E., Lewis, S., 2011. Yoga therapy as an adjunctive treatment for schizophrenia: a randomized, controlled pilot study. J. Altern. Complement. Med. 17 (7), 601-607. https://doi.org/10.1089/acm.2010.0075.

Westermann, S., Cavelti, M., Heibach, E., \& Caspar, F. (2015). Motive-oriented therapeutic relationship building for patients diagnosed with schizophrenia. Front. Psychol., 6 (September), 1294. https://doi.org/10.3389/fpsyg.2015.01294.

Wray, N. R., Ripke, S., Mattheisen, M., Trzaskowski, M., Byrne, E. M., Abdellaoui, A., ... Major Depressive Disorder Working Group of the Psychiatric Genomics Consortium. (2018). Genome-wide association analyses identify 44 risk variants and refine the genetic architecture of major depression. Nat. Genet., 50(5), 668-681. https://doi.org/ 10.1038/s41588-018-0090-3.

Zimbardo, P.G., 1999. Discontinuity theory: cognitive and social searches for rationality and normality may lead to madness. Adv. Exp. Soc. Psychol. 31 (C), 345-486. https://doi.org/10.1016/S0065-2601(08)60276-2. 